\title{
Perspective
}

\section{VDPV and polio end game strategy: the final frontier}

\author{
Dewesh Kumar $^{1 *}$, Neelesh Kapoor ${ }^{2}$, Pankaja Ravi Raghav ${ }^{1}$, Mahendra Singh Gehlot ${ }^{1}$
}

\author{
${ }^{1}$ Department of Community and Family Medicine, AIIMS, Jodhpur-342005, Rajasthan, India \\ ${ }^{2}$ NPSP-WHO, SRTL, Lucknow, UP, India
}

Received: 17 July 2014, Revised: 9 August 2014

Accepted: 13 August 2014

\author{
*Correspondence: \\ Dr. Dewesh Kumar, \\ E-mail: dr.dewesh@gmail.com
}

Copyright: (C) the author(s), publisher and licensee Medip Academy. This is an open-access article distributed under the terms of the Creative Commons Attribution Non-Commercial License, which permits unrestricted non-commercial use, distribution, and reproduction in any medium, provided the original work is properly cited.

\begin{abstract}
Polio eradication mission was impossible without the continual use of oral polio vaccine and it has set forth examples of its success all over the world. But the dynamics of OPV and low immunization coverage in some parts of world due to varied reasons is still a matter of concern due to the threat of Vaccine Derived Polio Virus (VDPV) and its circulation. The near eradication of poliomyelitis in whole world has intrigued the experts to control the VDPV and know more about their mutations and rising variations. Although their number are considerably less but they cannot be ignored and so there is a dire need to strategically implement the polio end game plan and rapid containment of newly found virus in any part of the world.
\end{abstract}

Keywords: Vaccine derived polio virus, Oral polio vaccine, Eradication

\section{INTRODUCTION}

Before world health assembly resolved to eradicate polio from the world in $1988,{ }^{1}$ many countries have achieved the status of polio free status owing to the use successful use of Oral Polio Vaccines (OPVs). The advantages of OPV were low cost; ease of administration; high vaccine efficacy for low number of doses; mucosal immunity to stop virus transmission; and vaccine-related virus spread contributing to "contact immunization". Based on the above mentioned reasons World Health Organization (WHO) considered Albert Sabin type OPV to be an ideal candidate for use in polio eradication. ${ }^{2}$ The success of OPVs is not overstated but the eradication of all viruses (wild as well as vaccine virus) as the end game plan seems to be a formidable task. The attenuated live virus of the Sabin type is genetically unstable ${ }^{3}$ and thus after prolonged reproduction in vaccines and their contacts, it tends to lose its attenuating mutations evolving into Vaccine-Derived Polio Viruses (VDPV) which have virtually no phenotypic distinctions from wild polioviruses and have the ability to circulate, especially in under-immunized communities, causing sporadic cases of the disease and outbreaks. ${ }^{4}$ Such VDPV are a serious threat to the global polio eradication initiative ${ }^{1}$ because the real eradication of polioviruses would not only include "true" wild viruses but also VDPV. 5

\section{VDPVs: WHAT IS IT?}

Poliovirus genome generally evolves at a rate of $1 \%$ per year. Hence the World Health Organization classifies VDPV as those Sabin vaccine derivatives in which the genome segment encoding the capsid protein VP1 deviates from the parental vaccine strain by at least $1 \%$ of nucleotides. ${ }^{6}$ Poliovirus isolates are grouped into three categories, based on the extent of divergence compared with the corresponding OPV(PVI, PV2 and PV3 strain): (1) Vaccine Related Polio Viruses (VRPVs) [<1\% divergent of (PV1 and PV3) strain or $<0.6 \%$ divergent (PV2)]; (2) VDPVs [VRPVs >1\% divergent (PV1 and PV3) or $>0.6 \%$ divergent (PV2)]; and 3) WPVs (WPV1, WPV2, and WPV3, no genetic evidence of derivation from any vaccine strain). ${ }^{7}$ VDPVs are further categorized $^{8}$ as: 
1) cVDPVs: In certain settings with very low immunization coverage, enough number of susceptible children exist providing an opportunity for the excreted vaccine derived polio virus to establish circulation in the community. These viruses are called Circulating Vaccine-Derived Polio Viruses (cVDPV). The level of population immunity is inversely proportional to the length of virus survival in the community. The proportional number of these cVDPVs according to the strains from 2000-14 are elucidated in Table 1.

2) iVDPVs: Prolonged vaccine derived viral survival and replication has been observed in small number of people with primary B-cell immunodefeciencies, who due to inadequate immune response fail to clear intestinal viral infection which otherwise doesn't take more than eight weeks to clear under normal circumstances. The occurrence of iVDPVs is very rare. Only 33 cases have been documented worldwide. Of these, most stopped excretion within six months or died.

3) aVDPVs: Since very little is known about this subtype and hence termed as ambiguous. Generally they are either clinical isolates from persons with no known immunodeficiency or are sewage isolates whose source could not be determined.

Table 1: Situational analysis of cVDPV (2000-2013).

\begin{tabular}{|ll|}
\hline cVDPV (type) & Frequency \\
\hline cVDPV type 1 & $79(11.02 \%)$ \\
\hline cVDPV type 2 & $625(87.17 \%)$ \\
\hline cVDPV type 3 & $13(1.81 \%)$ \\
\hline Total & $717(100 \%)$ \\
\hline
\end{tabular}

Although the frequency of occurrence of circulating vaccine derived poliovirus are rare but significant. A total of 23 cVDPV outbreaks have occurred between 20002013 resulting in 717 cases of VDPV and wild polio virus paralysed approximately 16200 children and during this period more than 10 billion doses of OPVs were given worldwide. The vaccine derived polio viruses are tested in global polio laboratory network using real-time reverse transcription-polymerase chain reaction (rRT-PCR) which targets nucleotide substitutions that occur in the emergence of the virus. Vaccine-derived polioviruses appear to be less transmissible than wild poliovirus. Circulating vaccine-derived polioviruses must be managed in the same way as wild poliovirus outbreaks. The solution is the same for all polio outbreaks: immunize every child several times with the oral vaccine to stop polio transmission, regardless of whether the virus is wild or vaccine-derived. Outbreaks are usually selflimiting or rapidly stopped with 2-3 rounds of highquality supplementary immunization activities. Once wild poliovirus transmission has been stopped globally, the vaccine-viruses will be the only source of live polioviruses in the community and could potentially lead to the re-emergence of polio. Use of the live polio vaccines in routine immunization programmes will therefore be phased out to eliminate the rare risks posed by vaccine-derived polioviruses.

\section{POLIO END GAME PLAN AND VDPV: CHALLENGES AND WAY FORWARD}

It was postulated quite early that cVDPVs will continue to circulate even 1-3 years after the eradication of WPVs. ${ }^{9}$ In the same light it was also realized that once the eradication of WPV is assured there will be an urgent need to stop using oral polio vaccine globally. The global Polio Eradication Initiative took valid cognizance of the matter and formulated its end game plan keeping in view of the impending challenge of cVDPVs.

Soon OPV has to be discontinued as early as feasible, for ethical reasons. When OPV is withdrawn, there will be a time overlap when children shedding vaccine viruses may transmit infection to immunity-naive infants and children, seeding the emergence of VDPV uninhibited by immunity. Such early lineages of VDPV will remain hidden in silent circulation until conditions are right for them to cause polio outbreaks. ${ }^{10}$ By then, their containment will be difficult. Thus, allowing the emergence and circulation of cVDPV is unwise and irresponsible. The emergence of VDPVs should be preempted using IPV. The elimination of VDPVs using IPV has been called phase 2 of polio eradication. For countries using OPV to eradicate WPVs, the need for a second phase is essential for the eradication of vaccine polioviruses.

The roadmap for phase 2 has been clearly defined in various scientific forums: introduce IPV in National Immunization Programme and achieve very high coverage and thereafter withdraw OPV nationally synchronously. ${ }^{11}$ In countries like India which was one of the last four endemic countries for Polio will soon be launching IPV in their immunization schedule as the introductory dose with the third dose of DPT and gradually inculcate with the four doses with DPT and withdrawal of OPVs simultaneously. The tempo of clinical (AFP) surveillance and virological investigation of every child with AFP will have to be continued until a minimum of three consecutive years after the total withdrawal of OPV and after the last poliovirus isolation, whichever is later. Routine vaccination with IPV alone should be used only in countries with high immunization coverage $(>90 \%)$ and at low risk of wild poliovirus importation and spread. A primary series of three IPV doses should be administered, beginning at 2 months of age. If the primary series begins earlier (e.g. with a 6-, 10 - and 14-week schedule), a booster dose should be administered after an interval of at least 6 months (fourdose IPV schedule). Some such countries may use a sequential schedule of IPV followed by OPV. Countries may have alternative schedules based on local epidemiology, including the documented risk of VAPP prior to 4 months of age. 
The plan thus seeks eventual withdrawal of oral polio vaccines from all 145 countries using it by 2019-2012. The first phase would seek withdrawal of type 2 antigen from tOPV. The end game plan seeks to replace OPV with Inactivated Polio Vaccine (IPV) finally. To effectively curtail the risk of cVDPVs post WPV eradication the switch from tOPV to bOPV and the eventual cessation of OPV use need to be coordinated and synchronized globally which in itself is quite a big challenge. A coordinated global response of such scale will require immaculate planning, logistics and programmatic bottleneck analysis and their timely resolution. But nevertheless the global eradication of Polio will be a great boon to mankind and would ensure an opportunity to live a better and healthier life to the most neglected children.

Funding: No funding sources Conflict of interest: None declared

Ethical approval: Not required

Disclaimer: The opinions expressed in this article are solely of the authors and should not be attributed to any institution/organization; they have been affiliated in the past or at present.

\section{REFERENCES}

1. Resolution WHA. 41.28. Polio eradication by the year 2000. In: Handbook of Resolutions and Decisions of the World Health Assembly and the Executive Board. 3rd ed. (1985-1992). Geneva Switzerland: WHO; 1993: 1-192.

2. Ghendon Y, Robertson SE. Interrupting the transmission of wild polioviruses with vaccine: immunological considerations. Bull WHO. 1994; $72: 873-83$.
3. Agol VI. Molecular mechanisms of poliovirus variation and evolution. Curr Top Microbiol Immunol. 2006;299:211-59.

4. Agol VI. Vaccine-derived polioviruses. Biologicals. 2006;34:103-8.

5. Ehrenfeld E, Glass RI, Agol VI, Chumakov K, Dowdle W, John TJ, et al. Polio immunization: moving forward. Lancet. 2008;371:1385-7.

6. Kew OM, Sutter RW, de Gourville EM, Dowdle WR, Pallansch WA. Vaccine-derived polioviruses and the endgame strategy for global polio eradication. Annu Rev Microbiol. 2005;59:587-635.

7. CDC. Update on vaccine-derived polioviruses worldwide, July 2009 - June 2011. Morb Mort Weekly Rep. 2011;60:846-50.

8. Polio Eradication. Vaccine derived polio virus (VDPV), 2014. Available at: http://www.polioeradication.org/Polioandprevention /Thevirus/Vaccinederivedpolioviruses.aspx. Accessed 3 April 2014.

9. Tebbens RJ, Pallansch MA, Kew OM, Carceres VM, Jafari H, Cochi SL, et al. Risks of paralytic disease due to wild or vaccine-derived poliovirus after eradication. Risk Anal. 2006;26:1471-505.

10. John TJ, Vashishtha VM. Eradication of vaccine viruses: why, when and how? Indian J Med Res. 2009;130:491-4.

11. John TJ. Polio eradication in India: what is the future? Indian Pediatr. 2003;40:455-62.

12. Polio Eradication. Polio eradication and end game strategic plan 2013-2018, 2014. Available at: http://www.polioeradication.org/Portals/0/Documen t/Resources/StrategyWork/PEESP_CH0_EN_US.pd f. Accessed 3 April 2014.

DOI: 10.5455/2349-3291.ijcp20140810

Cite this article as: Kumar D, Kapoor N, Raghav PR, Gehlot MS. VDPV and polio end game strategy: the final frontier. Int J Contemp Pediatr 2014;1:128-30. 